\title{
AFFINE SEMIGROUPS AND COHEN-MACAULAY RINGS GENERATED BY MONOMIALS
}

\author{
NGÓ VIÊT TRUNG AND LÊ TUÂN HOA
}

\begin{abstract}
We give a criterion for an arbitrary ring generated by monomials to be Cohen-Macaulay in terms of certain numerical and topological properties of the additive semigroup generated by the exponents of the monomials. As a consequence, the Cohen-Macaulayness of such a ring is dependent upon the characteristic of the ground field.
\end{abstract}

Introduction. Let $N$ denote the set of nonnegative integers. By an affine semigroup we mean a finitely generated submonoid $S$ of the additive monoid $N^{n}$, where $n$ is some positive integer. Let $k[S]$ denote the semigroup ring of $S$ over a field $k$. Then one can identify $k[S]$ with the subring of a polynomial ring $k\left[t_{1}, \ldots, t_{n}\right]$ generated by the monomials $t^{x}=t_{1}^{x_{1}} \cdots t_{n}^{x_{n}}, x=\left(x_{1}, \ldots, x_{n}\right) \in S$. Obviously, every subring of $k\left[t_{1}, \ldots, t_{n}\right]$ generated by a finite set of monomials is the semigroup ring of the affine semigroup in $N^{n}$ generated by the exponents of the monomials.

So one has, up to isomorphisms, a one-to-one correspondence between affine semigroups and affine varieties which are given parametrically by finite sets of monomials. When illustrating problems of algebraic geometry one almost inevitably tends to choose varieties of this type. Even when dealing with a quite general variety, either its singularities or a certain blowup may well be defined in local coordinates by monomials $[15,19]$. Moreover, one can also use rings generated by monomials to study solutions of linear equations in nonnegative integers or, equivalently, invariants of a torus acting linearly on a polynomial ring [11, 26]. Therefore, a criterion for such a ring to be Cohen-Macaulay in terms of the associated semigroup would be very useful. It should be mentioned that the first example of a non-CohenMacaulay domain (in modern language), given by F. S. Macaulay at the beginning of this century [17, p. 98], was the ring $k\left[t_{1}^{4}, t_{1}^{3} t_{2}, t_{1} t_{2}^{3}, t_{2}^{4}\right]$ and that analyzing this example, Gröbner [7] already posed the problem of classifying rings generated by monomials of the same degree with respect to their Cohen-Macaulayness.

The first step toward such a criterion was taken by Hochster [11], who succeeded in characterizing normal rings generated by monomials in terms of the associated semigroups and showed that they are always Cohen-Macaulay. Although this result was motivated by a conjecture on rings of invariants of reductive linear algebraic groups, which was later settled [12], its proof deserved much attention. It suggested

Received by the editors July 22, 1985.

1980 Mathematics Subject Classification (1985 Revision). Primary 13H10; Secondary 14M05.

(C)1986 American Mathematical Society $0002-9947 / 86 \$ 1.00+\$ .25$ per page 
the use of topological techniques in studying rings generated by monomials. The next step was a criterion given by Goto et al. for the case that the ring has a system of parameters consisting of monomials [5]. These results inspired many other works and were re-proved many times by different techniques, such as rational resolution [15], Hilbert functions of graded algebras [25], homology of polyhedral complexes $[13,26]$, and the Hodge algebra [10].

In both steps, one used the coincidence of an affine semigroup $S$ with one of its extensions to indicate the Cohen-Macaulayness of $k[S]$. Inspired by this phenomenon, Goto and Watanabe [6] defined a suitable extension $S^{\prime}$ of $S$ (see below) and claimed that $S^{\prime}=S$ is a necessary and sufficient condition for $k[S]$ to be CohenMacaulay.

Let $Z$ and $Q$ denote the sets of integers and rational numbers, respectively. Consider the elements of $S$ as points in the space $Q^{n}$. Let $G$ denote the additive group in $Z^{n}$ generated by $S$ and put $r=\operatorname{rank}_{Z} G$. Let $C_{S}$ denote the convex rational polyhedral cone spanned by $S$ in $Q^{n}$. Then $C_{S}$ is $r$-dimensional. Suppose that $F_{1}, \ldots, F_{m}$ are the $(r-1)$-dimensional faces of $C_{S}$. Let $S_{i}$ denote the set of elements $x \in G$ such that $x+y \in S$ for some element $y \in S \cap F_{i}, i=1, \ldots, m$. Then they define $S^{\prime}=\bigcap_{i=1}^{n} S_{i}$.

In this paper, we shall see that the condition $S^{\prime}=S$ is not sufficient for the Cohen-Macaulayness of $k[S]$, and that one has to add some topological condition on the convex cone $C_{S}$ to get a correct criterion. To formulate this we need some more notation.

Let $[1, m]$ denote the set of the integers $1, \ldots, m$. For every subset $J$ of $[1, m]$, set

$$
G_{J}=\bigcap_{i \notin J} S_{i} \backslash \bigcup_{j \in J} S_{j}
$$

and let $\pi_{J}$ be the simplicial complex of nonempty subsets $I$ of $J$ with the property $\bigcap_{i \in I} S \cap F_{i} \neq(0)$. Note that one calls $\pi_{J}$ acyclic if the reduced homology group $\tilde{H}_{q}\left(\pi_{J} ; k\right)$ vanishes for all $q \geqslant 0$.

MAIN THEOREM. Let $S$ be an arbitrary affine semigroup. Then $k[S]$ is a CohenMacaulay (resp. Gorenstein) ring if and only if the following conditions are satisfied:

(i) $S^{\prime}=S$ (resp. there exists an element $x \in G$ such that every element of $G_{[1, m]}$ is the difference of $x$ by some element of $S$ ).

(ii) For every nonempty proper subset $J$ of $[1, m], G_{J}=\varnothing$ or $\pi_{J}$ is acyclic.

It will follow from some property of the Cousin complex of $k[S]$ and from an explicit description of all local cohomology modules of $k\left[S^{\prime}\right]$ in terms of $G_{J}$ and $\pi_{J}$. We will also give some simple methods for checking the above conditions. As a consequence, one immediately gets the abovementioned results of Hochster and Goto et al. Another application is a criterion for the Rees algebra (blowing-up) of a ring generated by monomials to be Cohen-Macaulay (resp. Gorenstein). In particular, as in the work of Reisner on polynomial rings modulo ideals generated by square-free monomials [20] where a similar link to topology is given, we will show that the Cohen-Macaulayness of $k[S]$ is dependent upon the characteristic of the 
field $k$. Moreover, using the main theorem, we have been able to solve Gröbner's problem for some particular cases [31] (cf. [21, 29, and 30]).

We would like to mention that Stanley [26] also obtained a similar result on modules associated with solutions of systems of linear diophantine equations, which overlaps with ours only in Hochster's normal case.

This paper is organized in five sections. $\$ 1$ gives a counterexample to the result of Goto and Watanabe via some consideration on the Rees algebras of affine semigroup rings. In $\S 2, S^{\prime}$ will be related to the Cousin complex of $k[S]$ in order to show that $S^{\prime}=S$ if $k[S]$ is a Cohen-Macaulay ring. $\$ 3$ deals with the local cohomology modules of $k\left[S^{\prime}\right]$. Criteria for $k[S]$ to be Cohen-Macaulay (resp. Gorenstein) are given in $\$ 4$. There we will also deal with the Buchsbaumness of $k[S]$. The aim of $\S 5$ is to construct an affine semigroup ring whose local cohomology modules are just the reduced homology groups of a given finite simplicial complex.

All notations introduced above will be used throughout. Moreover, if $A$ and $B$ are subsets of $Z^{n}, G(A)$ denotes the additive group generated by $A$ in $Z^{n}$, and $A \pm B$ is the set of elements $a \pm b$ with $a \in A$ and $b \in B$. If $x, y, \ldots$ are elements of $Z^{n}$, we will denote their components by $x_{i}, y_{i}, \ldots, i=1, \ldots, n$, respectively. For unexplained notations and standard facts in commutative algebra, algebraic topology, and local cohomology, we refer the reader to [18, 24, and 8].

ACKNOWLEDGMENT. The authors would like to thank S. Ikeda for pointing out that our earlier conclusion on the Cohen-Macaulayness of the Rees algebras of Cohen-Macaulay rings generated by monomials is false (see $\$ 1$ ). This led us to check the result of $[6, \mathrm{II}]$. Thanks are also due to S. Goto for encouraging our study, and to L. Robbiano for some useful suggestions.

1. Counterexamples to the result of Goto and Watanabe. Let $S$ be an arbitrary affine semigroup in $N^{n}$. Set

$$
\begin{aligned}
\bar{S} & =\{x \in G ; p x \in S \text { for some } p>0\}, \\
S_{(i)} & =\left\{x \in S ; x_{i}=0\right\}, \quad i=1, \ldots, n .
\end{aligned}
$$

Then we call $S$ standard if the following conditions are satisfied:

(1) $\bar{S}=G \cap N^{n}$,

(2) $S_{(i)} \neq S_{(j)}$ for $i \neq j$,

(3) $\operatorname{rank}_{Z} G\left(S_{(i)}\right)=r-1, i=1, \ldots, n$.

Geometrically, these conditions mean that $C_{S}$ has exactly $n(r-1)$-dimensional faces lying on the hyperplane $x_{i}=0, i=1, \ldots, n$. In this case, we may assume that $S_{(i)}=S \cap F_{i}$ and $S_{i}=S-S_{(i)}$.

Goto and Watanabe [6, Theorem 3.3.3] claimed that if $S$ is standard, then $k[S]$ is a Cohen-Macaulay ring if and only if $S^{\prime}=S$. We shall see that this is false. First, we have to remark that every affine semigroup can be transformed isomorphically onto a standard one by the following technique which is due to Hochster [11, p. 323].

HOCHSTER'S TRANSFORMATION. Let $W$ denote the vector space generated by $S$ in $Q^{n}$. Then one can find $m$ linear functionals $l_{1}, \ldots, l_{m}$ from $W$ to $Q$ corresponding with $F_{1}, \ldots, F_{m}$ such that

$$
C_{S}=\left\{x \in W ; l_{i}(x) \geqslant 0 \text { for all } i\right\} .
$$


See also [15, p. 6]. Let $T$ denote the linear transformation which sends every element $x \in W$ to the element $\left(l_{1}(x), \ldots, l_{m}(x)\right) \in Q^{m}$. By replacing $l_{i}$ by a suitable positive integer multiple, one can assume that $T(S) \subseteq N^{m}$. Hochster has shown that $T(S)$ is isomorphic to $S$ and that

$$
\overline{T(S)}=G(T(S)) \cap N^{m}
$$

Obviously, $T$ also induces an isomorphism between the semigroups $S \cap F_{i}$ and $T(S)_{(i)}, i=1, \ldots, m$. Since $S \cap F_{i} \neq S \cap F_{j}$ for $i \neq j$, and $\operatorname{rank}_{Z} G\left(S \cap F_{i}\right)=r-$ 1 , we can conclude that $T(S)$ is a standard affine semigroup in $N^{m}$. Moreover, since

$$
T(S)_{i}=T(S)-T(S)_{(i)}=T\left(S-S \cap F_{i}\right)=T\left(S_{i}\right),
$$

we also have $T(S)^{\prime}=T\left(S^{\prime}\right)$.

According to this transformation, one can omit the assumption on the standardness of $S$ in the claim of Goto and Watanabe. The counterexample will also be constructed in the nonstandard case by using the following observation.

Let $\lambda: Q^{n} \rightarrow Q$ be a linear functional such that $\lambda(S) \subseteq N$, and, if $x \in S$ and $\lambda(x)=0$, then $x=0$. Then we call the affine semigroup

$$
E_{\lambda}:=\left\{(x, p) \in N^{n+1} ; x \in S \text { and } \lambda(x) \geqslant p\right\}
$$

a blowing-up extension of $S$. The name stems from the fact that one can define an $N$-grading on $k\left[E_{\lambda}\right]$ by setting $\operatorname{deg} t^{x}=\lambda(x)$ for all $x \in S[26$, p. 190] and that $k\left[E_{\lambda}\right]$ is isomorphic to the graded algebra $\oplus_{p=0}^{\infty} I_{p}$, where $I_{p}$ is the ideal of $k[S]$ generated by elements of degree $\geqslant p$.

LEMMA 1.1. Let $E_{\lambda}$ be a blowing-up extension of $S$. Then $E_{\lambda}^{\prime}=E_{\lambda}$ if and only if $S^{\prime}=S$.

Proof. Set $E=E_{\lambda}$. Then it is easy to see that $\operatorname{rank}_{Z} G(E)=r+1$ and $C_{E}$ has the following $m+2 r$-dimensional faces:

$$
P_{i}=\left\{(x, p) \in Q^{n+1} ; x \in F_{i} \text { and } \lambda(x) \geqslant p \geqslant 0\right\},
$$

$i=1, \ldots, m$, and

$$
\begin{aligned}
& P_{m+1}=\left\{(x, 0) \in Q^{n+1} ; x \in C_{S}\right\}, \\
& P_{m+2}=\left\{(x, p) \in Q^{n+1} ; x \in C_{S} \text { and } \lambda(x)=p\right\} .
\end{aligned}
$$

From this it follows that

$$
\begin{aligned}
E_{i} & =\left\{(x, p) \in Z^{n+1} ; x \in S_{i}\right\}, \quad i=1, \ldots, m, \\
E_{m+1} & =\left\{(x, p) \in Z^{n+1} ; x \in G \text { and } p \geqslant 0\right\}, \\
E_{m+2} & =\left\{(x, p) \in Z^{n+1} ; x \in G \text { and } \lambda(x) \geqslant p\right\} .
\end{aligned}
$$

Therefore, one can compute $E^{\prime}$ and get

$$
E^{\prime}=\left\{(x, p) \in Z^{n+1} ; x \in S^{\prime} \text { and } \lambda(x) \geqslant p \geqslant 0\right\} .
$$

As a consequence, $E^{\prime}=E$ if and only if $S^{\prime}=S$.

Now consider the case that $S$ can be generated by elements $x$ with $\lambda(x)=1$. Then $k\left[E_{\lambda}\right]$ is the Rees algebra $\oplus_{p=0}^{\infty} \underline{m}^{p}$, where $\underline{m}$ denotes the ideal of $k[S]$ generated by all elements $t^{x}, x \neq 0$. In this case, one knows that $k[E]$ being Cohen-Macaulay 
imposes certain conditions on $k[S]$, including $\underline{m}^{r}=q \underline{m}^{r-1}$ for every minimal reduction $\underline{q}$ of $\underline{m}[\mathbf{4}, \mathbf{1 4}]$. Note that an ideal $q$ of $k[S]$ is called a minimal reduction of $\underline{m}$ if $q$ is generated by $r$ elements whose initial forms in the associated graded ring $\oplus_{p=0}^{\infty} \underline{m}^{p} / \underline{m}^{p+1}$ form a homogeneous system of parameters of degree one, here $r=\operatorname{dim} k[S]$ because $\operatorname{rank}_{Z} G$ is equal to the transcendence degree of the fraction field of $k[S]$ over $k$.

EXAMPLE 1.2. Let $S$ be the affine semigroup in $N^{2}$ generated by the elements $(3,0),(2,1),(0,3)$. Then

$$
G=\left\{x \in Z^{2} ; x_{1}+x_{2} \equiv 0 \text { modulo } 3\right\} .
$$

Now one can rewrite

$$
S=\left\{x \in G \cap N^{2} ; x_{1} \neq 1\right\} .
$$

Using this formula, it is easy to check that $S$ is a standard affine semigroup with $S^{\prime}=S$. Obviously, $k[S]=k\left[t_{1}^{3}, t_{1}^{2} t_{2}, t_{2}^{3}\right]$ is a two-dimensional Cohen-Macaulay ring. Moreover, $q:=\left(t_{1}^{3}, t_{2}^{3}\right)$ is a minimal reduction of $\underline{m}:=\left(t_{1}^{3}, t_{1}^{2} t_{2}, t_{2}^{3}\right)$ and $\underline{m}^{2} \neq$ $\underline{q} \underline{m}$ because $t_{1}^{4} t_{2}^{2} \notin \underline{q} \underline{q}$. Let $\lambda$ be the linear functional $\left(x_{1}, x_{2}\right) \rightarrow\left(x_{1}+x_{2}\right) / 3$. Then $E_{\lambda}$ is the affine semigroup in $N^{3}$ generated by the elements $(3,0,0),(2,1,0),(0,3,0)$, $(3,0,1),(2,1,1),(0,3,1)$ and $k\left[E_{\lambda}\right]$ is isomorphic to the Rees algebra of $k[S]$ with respect to $\underline{m}$. By the above analysis, one gets $E_{\lambda}^{\prime}=E_{\lambda}$ but $k\left[E_{\lambda}\right]$ is not a Cohen-Macaulay ring. This yields a counterexample to the claim of Goto and Watanabe.

2. The meaning of $k\left[S^{\prime}\right]$. The aim of this section is to show that $S^{\prime}=S$ is a necessary condition for the Cohen-Macaulayness of $k[S]$ (the arguments of [6] do not work even for this conclusion). For this we have to use the notion of the Cousin complex of a commutative noetherian ring introduced by Sharp [22].

Recall that the Cousin complex $C(R)$ of a $Z^{n}$-graded commutative noetherian ring $R$ with identity is a complex of graded $R$-modules and graded homomorphisms

$$
0 \stackrel{d^{-2}}{\rightarrow} M^{-1}=R \stackrel{d^{-1}}{\rightarrow} M^{0} \stackrel{d^{0}}{\rightarrow} M^{1} \rightarrow \cdots \rightarrow M^{i} \stackrel{d^{i}}{\rightarrow} \cdots .
$$

It is defined inductively as follows: $M^{i}$ is the direct sum of the homogeneous localizations of coker $d^{i-2}$ at all graded prime ideals $\underline{p}$ of $R$ with ht $\underline{p}=i$, and $d^{i-1}$ is the composition of the canonical epimorphism $M^{i-1} \rightarrow \operatorname{coker} d^{i-2}$ and the homomorphism sending an element $x \in \operatorname{coker} d^{i-2}$ to the element $\oplus x / 1$ of $M^{i}$. Sharp [22] proved that $R$ is a Cohen-Macaulay ring if and only if $C(R)$ is exact. Although he only dealt with the nongraded case, his result can be easily extended to the $Z^{n}$-graded case as found in [6, I.\$4].

Now suppose that $R$ is a domain. Then $M^{0}=R_{(0)}$ and $M^{1}=\oplus\left(R_{(0)} / R_{p}\right)$, where $\underline{p}$ runs all graded prime ideals of $R$ with ht $\underline{p}=1$. Thus, $\operatorname{ker} d^{0}$ is the intersection of all homogeneous localizations $R_{p}$. Using this presentation of $\operatorname{ker} d^{0}$, we will prove the following

LEMma 2.1. Let $R=k[S]$. Then, with respect to the $Z^{n}$-graded structure of $k[S]$ inherited from $k\left[t_{1}, \ldots, t_{n}\right], \operatorname{ker} d^{0}=k\left[S^{\prime}\right]$.

This has some consequences for our further investigation. 
COROllary 2.2. $S^{\prime}=S$ if $k[S]$ is a Cohen-Macaulay ring.

Proof. Straightforward by the exactness of $C(k[S])$.

Corollary 2.3. $\operatorname{dim}\left(k\left[S^{\prime}\right] / k[S]\right)<\inf \left\{i ; k[S]_{\underline{p}}\right.$ is Cohen-Macaulay for all graded prime ideals $\underline{p}$ of $k[S]$ with ht $\underline{p}=r-i\} \leqslant r-1$.

Proof. By the stability of Cousin complexes under localizations [22, Theorem 3.5], $k\left[S^{\prime}\right]_{\underline{p}}=k[S]_{\underline{p}}$ and therefore $\left(k\left[S^{\prime}\right] / k[S]\right)_{\underline{p}}=0$ for all graded prime ideals $\underline{p}$ of $k[S]$ such that $k[S]_{p}$ is Cohen-Macaulay. In particular, this condition is always satisfied if ht $\underline{p}=1$. Hence the statement is immediate.

REMARK. We would like to mention that the Cousin complex $C(R)$ carries much information on the local cohomology of $R$ [23]. Since $C\left(\operatorname{ker} d^{0}\right)$ differs from $C(R)$ only at the first term, i.e. $C\left(\operatorname{ker} d^{0}\right)$ has the form

$$
0 \rightarrow \operatorname{ker} d^{0} \rightarrow M^{0} \stackrel{d^{0}}{\rightarrow} M^{1} \rightarrow \cdots \rightarrow M^{i} \rightarrow \cdots
$$

(which can be easily checked), one can use $\operatorname{ker} d^{0}$ as an approximation of $R$ in studying local cohomology and related subjects as shown above for $k[S]$.

To prove Lemma 2.1 we have to determine all homogeneous localizations of $k[S]$ at graded prime ideals of height one. This can be done purely in terms of $S$ as follows.

Let $A$ be an arbitrary subset of $G$. Then we will denote by $k[A]$ the $k$-vector space spanned by $A$ in $k[G]$. If $A+S \subseteq A$, we call $A$ an $S$-ideal. In this case, $k[A]$ can be considered as a $Z^{n}$-graded module over $k[S]$. If $A=B \backslash C$, where $B \supseteq C$ are $S$-ideals, $k[A]$ will be identified with the factor module $k[B] / k[C]$.

We call a proper subset $P$ of $S$ a prime ideal if $P$ is an $S$-ideal and $S \backslash P$ is additively closed. It is easily seen that every graded prime ideal $\underline{p}$ of $k[S]$ is exactly of the form $k[P]$ for some prime ideal $P$ of $S$ and that the homogeneous localization $k[S]_{\underline{p}}$ is isomorphic to $k[S-(S \backslash P)]$.

LEMMA 2.4. For every subset $I$ of $[1, n]$, set

$$
P_{I}= \begin{cases}(0) & \text { if } I=\varnothing, \\ \left\{x \in S ; x_{i}>0 \text { for some } i \in I\right\} & \text { if } I \neq \varnothing .\end{cases}
$$

If $S$ is a standard affine semigroup, then $\left\{P_{I}\right\}$ is the set of prime ideals of $S$.

Proof. Obviously, every $P_{I}$ is an $S$-ideal and

$$
S \backslash P_{I}=\left\{x \in S ; x_{i}=0 \text { for all } i \in I\right\}
$$

is additively closed. Hence $P_{I}$ is a prime ideal of $S$. Conversely, let $P$ be an arbitrary prime ideal of $S$. Let $I$ be the set of integers $i \in[1, n]$ such that

$$
P_{i}:=\left\{x \in S ; x_{i}>0\right\}
$$

is contained in $P$. Then $P_{I}=\bigcup_{i \in I} P_{i} \subseteq P$. If $I=[1, n], P_{I}=S \backslash(0)$, hence $P=P_{I}$. If $I \subset[1, n]$ properly, choose an element $x \in P_{i} \backslash P$ for every $i \in[1, n] \backslash I$. Let $y$ denote the sum of these elements. Then $y_{i}>0$ for all $i \notin I$. Therefore, if $P$ contains an element $z \notin P_{I}$, i.e. $z_{i}=0$ for $i \in I$, one can find a positive integer $p$ such that 
$p y_{i}-z_{i} \geqslant 0$ for all $i \in[1, n]$. From this it follows that $p y-z \in G \cap N^{n}=\bar{S}$. Hence $q(p y-z) \in S$ for some $q>0$. Since $p q y=q z+q(p y-z) \in P+S \subseteq P$, one gets $y \in P$ because $S \backslash P$ is additively closed. Therefore, by the same reason, some of the chosen elements $x$ must belong to $P$, a contradiction. So $P=P_{I}$, as required.

Lemma 2.1 was already known in the normal case [6, 3.1.5].

Proof of LeMma 2.1. Via Hochster's transformation (\$1) we may assume that $S$ is standard. In this case, one can set $S_{i}=S-S_{(i)}, i=1, \ldots, n$. Since $S_{(i)}=S \backslash P_{i}$,

$$
S^{\prime}=\bigcap_{i=1}^{n}\left(S-\left(S \backslash P_{i}\right)\right) \text {. }
$$

Put $\underline{p}_{i}=k\left[P_{i}\right]$. Then

$$
k\left[S^{\prime}\right]=\bigcap_{i=1}^{n} k[S]_{\underline{p}_{i}} .
$$

It remains to show that $\left\{\underline{p}_{i}\right\}$ is the set of graded prime ideals of height one of $k[S]$. By Lemma 2.4, we only need to show that ht $\underline{p}_{i}=1$ or, equivalently, $\operatorname{dim} k[S] / \underline{p}_{i}=$ $r-1$ for all $i=1, \ldots, n$. Since $k[S] / \underline{p}_{i}=k\left[S \backslash P_{i}\right]=k\left[S_{(i)}\right]$, this is immediate because $r-1=\operatorname{rank}_{Z} G\left(S_{(i)}\right)$ is the transcendence degree of the fraction field of $k\left[S_{(i)}\right]$ over $k$.

3. Local cohomology of $k\left[S^{\prime}\right]$. Given two $Z^{n}$-graded modules $M_{1}$ and $M_{2}$ over $k[S]$, one can define the Segre product

$$
M_{1} \otimes M_{2}:=\bigoplus_{x \in Z^{n}}\left[M_{1}\right]_{x} \otimes_{k}\left[M_{2}\right]_{x}
$$

where $\left[M_{1}\right]_{x}$ and $\left[M_{2}\right]_{x}$ denote the $x$-graded piece of $M_{1}$ and $M_{2}$. Obviously, $M_{1} \otimes M_{2}$ can be considered as a $Z^{n}$-graded module over $k[S]=k[S] \underline{\otimes} k[S]$. Note that

$$
k[A] \underline{\otimes} k[B]=k[A \cap B]
$$

if $A$ and $B$ are $S$-ideals in $G$.

Let $D_{i}^{*}$ denote the complex

$$
0 \rightarrow D_{i}^{0} \stackrel{d}{\rightarrow} D_{i}^{1} \rightarrow 0
$$

where $D_{i}^{0}:=k[G], D_{i}^{1}:=k\left[G \backslash S_{i}\right]$, and $d$ is the canonical map from $k[G]$ to $k\left[G \backslash S_{i}\right]=k[G] / k\left[S_{i}\right], i=1, \ldots, m$. Put

$$
D^{*}:=D_{i}^{i} \underline{\otimes} \cdots \underline{\otimes} D_{m}^{.}
$$

Lemma 3.1. $D^{*}$ is a coresolution of $k\left[S^{\prime}\right]$.

Proof. Note that $H^{0}\left(D_{i}^{*}\right)=k\left[S_{i}\right]$ and $H^{p}\left(D_{i}^{*}\right)=0$ for all $p \neq 0, i=1, \ldots, m$. Then, using the Künneth formula [13, Chapter V, (10.1)], one has

$$
\begin{aligned}
& H^{0}\left(D^{*}\right)=k\left[S_{1}\right] \underline{\otimes} \cdots \underline{\otimes}\left[S_{m}\right]=k\left[\bigcap_{i=1}^{m} S_{i}\right]=k\left[S^{\prime}\right], \\
& H^{p}\left(D^{*}\right)=0 \text { for all } p \neq 0,
\end{aligned}
$$

which yields the statement. 
By the definition of $D$; we can write

$$
D^{p}=\bigoplus_{\substack{I \subseteq[1, m] \\ \# I=p}} D_{I}
$$

for all $p \geqslant 0$, where $D_{I}$ is the Segre product of the modules $D_{i}^{1}$ with $i \in I$.

Let $\pi$ be the simplicial complex of nonempty subsets $J$ of $[1, m]$ with the property $\bigcap_{i \in J} S \cap F_{i} \neq(0)$. Let $D_{\pi}^{\cdot}$ denote the subcomplex of $D^{\circ}$ consisting of the terms

$$
D_{\pi}^{p}:=\bigoplus_{\substack{I \in \pi \\ \# I=p}} D_{I},
$$

$p \geqslant 0$. Then we have the following preliminary formula for the local cohomology modules of $k\left[S^{\prime}\right]$ with respect to $\underline{m}:=k[S \backslash(0)]$.

Lemma 3.2 [6, CoRollary 3.3.7]. $H_{\underline{m}}^{q}\left(k\left[S^{\prime}\right]\right)=H^{q}\left(D^{*} / D_{\pi}^{*}\right)$ for all $q \geqslant 0$.

Since the proof of [6, Corollary 3.3.7] is not clear enough (some arguments do not work) and to make this paper self-contained, we will do it once more.

Proof of Lemma 3.2. By Lemma 3.1, one need only show that $H_{\underline{m}}^{0}\left(D^{\circ}\right)=D^{*} / D_{\pi}^{*}$ and $H_{\underline{m}}^{q}\left(D^{\circ}\right)=0$ for all $q>0$. In terms of $D_{I}$, this means

(1) $H_{m}^{q}\left(D_{I}\right)=0$ for all $q \geqslant 0$ if $I \in \pi$,

(2) $H_{\underline{m}}^{0}\left(D_{I}\right)=D_{I}$ and $H_{\underline{m}}^{q}\left(D_{I}\right)=0$ for all $q>0$ if $I \notin \pi$.

To prove these facts we may assume that $S$ is a standard affine semigroup by using Hochster's transformation $(\$ 1)$. Moreover, we want to note that by definition,

$$
D_{I}=k\left[G \backslash \bigcup_{i \in I} S_{i}\right]
$$

for all subsets $I$ of $[1, n]$.

To (1). Let $x \neq(0)$ be an arbitrary element of $\bigcap_{i \in I} S_{(i)}$. Since $S_{(i)}$ is additively closed,

$$
S_{i}-x=S-S_{(i)}-x \subseteq S-S_{(i)}=S_{i}
$$

for all $i \in I$. Therefore, if $x+y \in \cup_{i \in I} S_{i}$ for some element $y \in G$, then $y \in$ $\bigcup_{i \in I} S_{i}$ too. From this it follows that the multiplication map by $t^{x}$ is bijective on $D_{I}$ and hence on $H_{\underline{m}}^{q}\left(D_{I}\right)$ for all $q \geqslant 0$. Since every element of $H_{\underline{m}}^{q}\left(D_{I}\right)$ is annihilated by some power of $t^{x}, H_{m}^{q}\left(D_{I}\right)=0$, as required.

To (2). It suffices to show that every element of $D_{I}$ is annihilated by some power of $\underline{m}$ or, equivalently, that for any pair of elements $x \in G$ and $y \in S \backslash(0)$, there exist $i \in I$ and $p>0$ such that $x+p y \in S_{i}$. First, since $y \neq(0)$ and $\bigcap_{i \in I} S_{(i)}=(0)$, there exists $i \in I$ such that $y_{i}>0$. For this $i$, one has $\sup \left\{x_{i}+p y_{i} ; p>0\right\}=\infty$. Therefore, one only need to show that there is a bound for the $i$ th components of elements of $G \backslash S_{i}$. For this purpose, choose an element $z \in S \backslash S_{(i)}$. Let $H$ denote the additive group generated by $S_{(i)}$ and $z$ in $Z^{n}$. Since $\operatorname{rank}_{Z} G\left(S_{(i)}\right)=r-1$, $\operatorname{rank}_{Z} H=r$. Thus, one can find a finite set of elements of $S$ such that $G$ can be obtained from $A$ by translating along $H$, that is $G=A+H$. Note that

$$
H=\bigcup_{m \in Z}\left(G\left(S_{(i)}\right)+m z\right), \quad \bigcup_{m>0}\left(A+G\left(S_{(i)}\right)+m z\right) \subseteq S_{i} .
$$


Then one gets

$$
G \backslash S_{i} \subseteq \bigcup_{m \in N}\left(A+G\left(S_{(i)}\right)-m z\right) .
$$

Hence $\max \left\{u_{i} ; u \in A\right\}$ bounds the $i$ th components of elements of $G \backslash S_{i}$. The proof of Lemma 3.2 is now complete.

Now, we shall see that every graded piece $\left[H_{m}^{q}\left(k\left[S^{\prime}\right]\right)\right]_{x}$ can be expressed completely in terms of some simplicial subcomplex $\pi_{J}$ of $\pi$. Note that for every subset $J$ of $[1, m], \pi_{J}$ is the simplicial complex of nonempty subsets $I$ of $J$ with the property $\bigcap_{i \in I} S \cap F_{i} \neq(0)$ (see Introduction). Since $\left[H_{\underline{m}}^{q}\left(k\left[S^{\prime}\right]\right)\right]_{x}=0$ for all $x \notin G$, we may restrict our attention to elements $x \in G$. For such $x$ set

$$
J_{x}:=\left\{i \in[1, m] ; x \notin S_{i}\right\} .
$$

Then the main result of this section can be formulated as follows.

THEOREM 3.3. $\left[H_{\underline{m}}^{q}\left(k\left[S^{\prime}\right]\right)\right]_{x}=\tilde{H}_{q-2}\left(\pi_{J_{x}} ; k\right)$ for all $q \geqslant 0$.

Proof. For simplicity, set $J=J_{x}$. Let $I$ be an arbitrary subset of $[1, m]$. Note that $D_{I}=k\left[G \backslash \bigcup_{i \in I} S_{i}\right]$. Then we have the following formula:

$$
\left[D_{I}\right]_{x}= \begin{cases}k & \text { if } I \subseteq J, \\ 0 & \text { if } I \nsubseteq J .\end{cases}
$$

Let $\Delta_{J}$ denote the simplicial complex of all nonempty subsets of $J$. Let $C\left(\Delta_{J}\right)$ be the oriented chain complex of $\Delta_{J}$ (see [24]). Then one can consider $\left[D^{\circ}\right]_{x}$ as the complex $\operatorname{Hom}\left(C\left(\Delta_{J}\right), k\right)$ with degree shifted by one. Therefore, by Lemma 3.1,

$$
\left[H_{\underline{m}}^{q}\left(k\left[S^{\prime}\right]\right)\right]_{x}=H^{q}\left(\left[D \cdot D_{\pi}^{\cdot}\right]_{x}\right)=H^{q-1}\left(\Delta_{J}, \Delta_{J} \cap \pi ; k\right) .
$$

Since $\tilde{H}^{q}\left(\Delta_{J} ; k\right)=0$ for all $q$, from the reduced cohomology sequence of the pair $\Delta_{J}$ and $\Delta_{J} \cap \pi$ one gets

$$
H^{q-1}\left(\Delta_{J}, \Delta_{J} \cap \pi ; k\right)=\tilde{H}^{q-2}\left(\Delta_{J} \cap \pi ; k\right) .
$$

Note that $\Delta_{J} \cap \pi=\pi_{J}$ and that cohomology and homology coincide over a field. Then one can conclude that

$$
\left[H_{\underline{m}}^{q}\left(k\left[S^{\prime}\right]\right)\right]_{x}=\tilde{H}_{q-2}\left(\pi_{J} ; k\right) .
$$

Corollary 3.4. (i) $H_{\underline{m}}^{1}\left(k\left[S^{\prime}\right]\right)=0$.

(ii) $H_{m}^{1}(k[S])=k\left[S^{\prime \prime} \backslash S\right]$, where $S^{\prime \prime}$ denotes the set of all elements $x \in G$ such that $x+p(S \backslash(0)) \subseteq S$ for some positive integer $p$.

Proof. (i) is immediate because $\tilde{H}_{-1}\left(\pi_{J_{x}} ; k\right)=0$ for every $x \in G$. For (ii), consider the exact sequence

$$
0 \rightarrow k[S] \rightarrow k\left[S^{\prime}\right] \rightarrow k\left[S^{\prime} \backslash S\right] \rightarrow 0 .
$$

Since $H_{\underline{m}}^{i}\left(k\left[S^{\prime}\right]\right)=0, i=0,1$, we get $H_{\underline{m}}^{1}(k[S]) \cong H_{\underline{m}}^{0}\left(k\left[S^{\prime} \backslash S\right]\right)$. But $H_{\underline{m}}^{0}\left(k\left[S^{\prime} \backslash S\right]\right)$ is the set of all elements $f \in k\left[S^{\prime} \backslash S\right]$ which are annihilated by $\underline{m}^{p}$ for some $p \geqslant 0$. Translating this into the language of semigroups, we get $H_{\underline{m}}^{0}\left(k\left[S^{\prime} \backslash S\right]\right)=k\left[S^{\prime \prime} \backslash S\right]$.

In order to derive other consequences of Theorem 3.3 we have to replace $\pi_{J}$ by a more tractable object. To this end, let $P$ be a nondegenerate hyperplane section of $C_{S} . P$ is a $(r-1)$-dimensional convex polytope with $m(r-2)$-dimensional faces. 
Let $P^{*}$ denote the dual polytope of $P$ [9]. Then there is a one-to-one inclusion reversing correspondence $F \leftrightarrow F^{*}$ between faces $F$ of $P$ and $F^{*}$ of $P^{*}$ such that $\operatorname{dim} F+\operatorname{dim} F^{*}=r-2$. So $P^{*}$ has $m$ vertices. Let $v_{i}$ be the vertex of $P^{*}$ corresponding with the $(r-2)$-dimensional face of $P$ on $F_{i}, i=1, \ldots, m$. It is not hard to realize that $\pi_{J}$ is the simplicial complex of nonempty subsets $I \subseteq J$ such that there is a proper face of $P^{*}$ containing all vertices $v_{i}$ with $i \in I$. Let $P_{J}^{*}$ denote the union of all convex polytopes on $\partial P^{*}$ spanned by a set of vertices $v_{i}$ with $i \in I$ for some maximal $I$ of $\pi_{J}$.

LEMMA 3.5. $P_{J}^{*}$ and the geometric realization $\left|\pi_{J}\right|$ have the same homotopy type.

Proof. We shall prove a more general statement. Let $U$ be a finite union of convex rational polytopes in $Q^{n}$ such that the intersection of two polytopes of $U$ is either empty or a proper common face. Suppose that $v_{1}, \ldots, v_{s}$ are the vertices of the polytopes of $U$. Let $\pi(U)$ denote the simplicial complex of nonempty subsets $I$ of $[1, s]$ such that there is a polytope of $U$ which contains all vertices $v_{i}$ with $i \in I$. Then there exists a canonical linear map $f$ from $|\pi(U)|$ onto $U$. We claim that $f$ is a homotopy equivalence. That is trivial if $U$ consists of only one polytope because $|\pi(U)|$ and $U$ are contractible spaces in this case. If $U$ consists of more than one polytope, we take a polytope $u$ out of $U$. Let $V$ denote the union of the remaining polytopes. By induction, we may assume that $f$ induces a homotopy equivalence between $|\pi(u)|,|\pi(V)|,|\pi(u \cap V)|$ and $u, V, u \cap V$, respectively. Therefore, $f$ must be a homotopy equivalence between $|\pi(U)|=|\pi(u)| \cup|\pi(V)|$ and $U=u \cup V$, as claimed. Since $\pi_{J}=\pi\left(P_{J}^{*}\right)$, this yields the statement.

Corollary 3.6.

(i)

$$
\tilde{H}_{q-2}\left(\pi_{[1, m]} ; k\right)= \begin{cases}0 & \text { if } q \neq r \\ k & \text { if } q=r .\end{cases}
$$

(ii) If $J \neq[1, m], \tilde{H}_{q-2}\left(\pi_{J} ; k\right)=0$ for $q \geqslant r$.

(iii) If $J \in \pi$ or $\# J=m-1, \tilde{H}_{q-2}\left(\pi_{J} ; k\right)=0$ for all $q$.

Proof. By Lemma 3.5, we may replace any $\pi_{J}$ by $P_{J}^{*}$. Then (i) follows from the fact that $P_{[1, m]}^{*}=\partial P^{*}$ is homeomorphic to a $(r-2)$-dimensional sphere. Since every proper subspace of $\partial P^{*}$ contains no $(r-2)$-dimensional "hole", (ii) is also immediate. In particular, if $J \in \pi$ or $\# J=m-1, P_{J}$ is homeomorphic to a ball, hence (iii).

Now we will combine Theorem 3.3 with Corollary 3.6 to get some description of $H_{m}^{i}\left(k\left[S^{\prime}\right]\right), i \geqslant 2$.

First, we would like to remark that Theorem 3.3 divides $G$ into classes of points $x$ with the same $J_{x}$. It is easily seen that these classes are exactly the sets

$$
G_{J}:=\bigcap_{i \notin J} S_{i} \backslash \bigcup_{j \in J} S_{j}
$$

introduced in the Introduction. 
COROLlaRy 3.7. For all $q<r$, there is an isomorphism of $k$-vector spaces

$$
H_{\underline{m}}^{q}\left(k\left[S^{\prime}\right]\right) \cong \bigoplus_{\substack{J \notin \pi \\ \# J \leqslant m-2}} k\left[G_{J}\right] \otimes_{k} \tilde{H}_{q-2}\left(\pi_{J} ; k\right) .
$$

Proof. By Corollary 3.6, $\tilde{H}_{q-2}\left(\pi_{J} ; k\right)=0$ if $J \in \pi$ or $\# J=m, m-1$. Therefore, by Theorem 3.3 , taking the sum of all $H_{q-2}\left(\pi_{J_{x}} ; k\right)$ over $x \in G$ will yield the above formula for $H_{\underline{m}}^{q}\left(k\left[S^{\prime}\right]\right)$.

REMARK. We are unable to determine the module structure of the above presentation of $H_{\underline{m}}^{q}\left(k\left[S^{\prime}\right]\right), q<r$.

COROLLARY 3.8. $H_{\underline{m}}^{r}(k[S]) \cong H_{\underline{m}}^{r}\left(k\left[S^{\prime}\right]\right) \cong k\left[G_{[1, m]}\right]$.

Proof. First, consider the exact sequence

$$
0 \rightarrow k[S] \rightarrow k\left[S^{\prime}\right] \rightarrow k\left[S^{\prime} \backslash S\right] \rightarrow 0 .
$$

Note that $\operatorname{dim} k\left[S^{\prime} \backslash S\right] \leqslant r-2$ by Corollary 2.3. Then one gets $H_{\underline{m}}^{r}(k[S]) \cong$ $H_{\underline{m}}^{r}\left(k\left[S^{\prime}\right]\right)$. By Theorem 3.3 and Corollary 3.6,

$$
\left[H_{\underline{m}}^{r}\left(k\left[S^{\prime}\right]\right)\right]_{x}= \begin{cases}0 & \text { if } x \notin G_{[1, m]} \\ k & \text { if } x \in G_{[1, m]}\end{cases}
$$

Therefore, using Lemma 3.2, it is not hard to see that

$$
H_{\underline{m}}^{r}\left(k\left[S^{\prime}\right]\right) \cong k\left[G_{[1, m]}\right] .
$$

EXAMPLE 3.9. Let $S$ be the affine semigroup in $N^{4}$ generated by the elements $(3,0,0,3),(2,1,0,3),(0,3,0,3),(3,0,1,0),(2,1,1,0),(0,3,1,0)$. It is easily seen that $S$ is isomorphic to the affine semigroup $E_{\lambda}$ of Example 1.2. Note that

$$
G=\left\{x \in Z^{4} ; x_{1}+x_{2}=3 x_{3}+x_{4} \equiv 0 \text { modulo } 3\right\} .
$$

Then one can write

$$
S=\left\{x \in G \cap N^{4} ; x_{1} \neq 1\right\}
$$

From this it follows that

$$
\bar{S}=G \cap N^{4}, \quad G\left(S^{(i)}\right)=\left\{x \in G ; x_{i}=0\right\}
$$

for $i=1,2,3,4$. Hence $S$ is a standard affine semigroup. Now one can identify $S_{i}$ with $S-S_{(i)}$ and get

$$
\begin{aligned}
& S_{1}=\left\{x \in G ; x_{1} \geqslant 0, x_{1} \neq 1\right\}, \\
& S_{i}=\left\{x \in G ; x_{i} \geqslant 0\right\}, \quad i=2,3,4,
\end{aligned}
$$

which implies $S^{\prime}=S$. Obviously, $\pi$ consists of the sets $\varnothing,\{1\},\{2\},\{3\},\{4\},\{1,3\}$, $\{1,4\},\{2,3\},\{2,4\}$. Hence $P^{*}$ is quadrilateral:

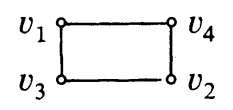

There are only two sets $J=\{1,2\},\{3,4\}$ with the properties $J \notin \pi$ and $|J| \leqslant 2$. Since the corresponding simplicial complex $\pi_{J}$ consists of two points,

$$
\tilde{H}_{q}\left(\pi_{J} ; k\right)= \begin{cases}k & \text { if } q=0 \\ 0 & \text { if } q \neq 0\end{cases}
$$


On the other hand, $G_{\{1,2\}}=\{(1,-1,0,0)\}, G_{\{3,4\}}=\varnothing$. Summing up, one gets

$$
H_{\underline{m}}^{q}(k[S])= \begin{cases}0 & \text { if } q \neq 2,3, \\ k & \text { if } q=2, \\ k\left[G_{[1,4]}\right] & \text { if } q=3,\end{cases}
$$

where $H_{\underline{m}}^{2}(k[S])$ is concentrated in degree $(1,-1,0,0) \in S_{3} \cup S_{4}$. This yields a counterexample to the formula $\left[H_{m}^{q}\left(k\left[S^{\prime}\right]\right)\right]_{x}=0, x \in \bigcup_{i=1}^{m} S_{i}$, given in [6, Lemma 3.3.8]. Actually, this was the critical point leading to the mistake of [6, Theorem 3.3.3].

4. Cohen-Macaulayness of $k[S]$. Now we are going to prove the Main Theorem of the Introduction. According to Corollary 3.6, it can be reformulated as follows.

THEOREM 4.1. $k[S]$ is Cohen-Macaulay (resp. Gorenstein) if and only if the following conditions are satisfied:

(i) $S^{\prime}=S$ (resp. $G_{[1, m]}=x-S$ for some $\left.x \in G\right)$,

(ii) For every nonempty subset $J$ of $[1, m]$ with $J \notin \pi$ and $\# J \leqslant m-2, G_{J}=\varnothing$ or $\tilde{H}_{q}\left(\pi_{J} ; k\right)=0$ for all $q<r-2$.

Proof. Concerning the Cohen-Macaulayness of $k[S]$, we may assume that $S^{\prime}=S$ by using Corollary 2.2. In this case we may apply Corollary 4.7 and get the statement because $k[S]$ is Cohen-Macaulay if and only if $H_{m}^{q}(k[S])=0$ for all $q<r$ [8]. It is also known that $k[S]$ is Gorenstein if and only if $k[S]$ is CohenMacaulay and its canonical module

$$
K:=\operatorname{Hom}_{k}\left(H_{\underline{m}}^{r}(k[S]), k\right)
$$

is isomorphic to $k[S]$ with degree shifted by some $x \in G$, see [6, Corollary 2.2.3 or 25, Theorem 6.1]. Set $C=G_{[1, m]}$. By Corollary 3.8, $H_{\underline{m}}^{r}(k[S]) \cong k[C]$. Hence $K \cong k[-C]$. Therefore, it suffices to show that the condition $C=x-S$ implies $S^{\prime}=S$. Let $y$ be an arbitrary element of $S^{\prime}$. If $y \notin S, x-y \notin C$. Thus, $x-y \in S_{i}$ for some $i \in[1, m]$. Since $y \in S^{\prime} \subseteq S_{i}, x \in S_{i}+y \subseteq S_{i}$ too. From this it follows that $x \notin C=x-S$, a contradiction.

REMARK. In Theorem 4.1, one can replace $\pi_{J}$ by the simplicial complex

$$
\pi_{J}^{*}:=\{I \subseteq J ; J \backslash I \notin \pi\} .
$$

For, using the one-to-one correspondence $I \leftrightarrow J \backslash I$ between faces of $\pi_{J}$ and $\pi_{J}^{*}$, one can check that

$$
\tilde{H}_{q}\left(\pi_{J}^{*} ; k\right)=H^{s-q}\left(\Delta_{J}, \pi_{J} ; k\right)=\tilde{H}_{s-q-1}\left(\pi_{J} ; k\right),
$$

where $\Delta_{J}$ is the simplicial complex of all nonempty subsets of $J$ and $s$ is the number of elements of $J$.

In the following, we will give an affine semigroup which satisfies condition (i) of Theorem 4.1 but not condition (ii).

EXAMPLE 4.2. Suppose that

$$
\begin{aligned}
& G=\left\{x \in Z^{4} ; x_{1}+x_{2}=x_{3}+x_{4}\right\}, \\
& S=\left\{x \in G \cap N^{4} ; x_{1} \neq 1, x_{3} \neq 1\right\} .
\end{aligned}
$$


It is not hard to see that $S$ is a standard affine semigroup. Therefore, one can set $S_{i}=S-S_{(i)}$ and get

$$
S_{i}= \begin{cases}\left\{x \in G ; x_{i} \geqslant 0, x_{i} \neq 1\right\}, & i=1,3, \\ \left\{x \in G ; x_{i} \geqslant 0\right\}, & i=2,4 .\end{cases}
$$

From this it follows that

$$
\begin{aligned}
G_{[1,4]} & =\left\{x \in G ; x_{i}<0 \text { or } x_{i}=1 \text { for } i=1,3, x_{i}<0 \text { for } i=2,4\right\} \\
& =(1,-1,1,-1)-S .
\end{aligned}
$$

Hence condition (i) of Theorem 4.1 is satisfied. On the other hand, it is easily seen that $\pi$ consists of the sets $\varnothing,\{1\},\{2\},\{3\},\{4\},\{1,3\},\{1,4\},\{2,3\},\{2,4\}$. For $J=\{1,2\}$ or $\{3,4\}, \pi_{J}$ consists of two points. Since $G_{\{1,2\}}=\{(0,0,1,-1)\}$ and $G_{\{3,4\}}=\{(1,-1,0,0)\}$, condition (ii) of Theorem 4.1 is not satisfied. Therefore, by Corollary $3.7, k[S]$ is not Cohen-Macaulay. This yields a counterexample to [6, Theorem 3.3.3] where one also gave a false criterion for the Gorensteinness of $k[S]$ containing only the condition $G_{[1, m]}=x-S$.

To check condition (i) of Theorem 4.1 one should make use of the following notation.

Let $A$ be a finite set of elements of $S \backslash(0)$ such that $A$ spans the convex cone $C_{S}$. Then we will denote by $S_{A}$ the set of elements $x \in G$ such that $x+y \in S$ for all elements $y$ of some minimal generic subset of $A$, where we call a subset $B$ of $S$ generic if $B \cap F_{i} \neq \varnothing$ for all $i=1, \ldots, m$.

LEMMA 4.3. $S^{\prime}=S$ if and only if $S_{A}=S$.

Proof. Since $S \subseteq S_{A} \subseteq S^{\prime}$, it suffices to show that $S_{A}=S$ implies $S^{\prime}=S$. Without restriction, we may assume that $S$ is standard (\$1). Let $x$ be an arbitrary element of $S^{\prime}$. Then for every $i \in[1, n]$, one can find an element $y^{(i)} \in S_{(i)}$ such that $x+y^{(i)} \in S$. Since $A$ spans $C_{S}$, one may assume that

$$
y^{(i)}=\sum_{z \in A \cap S_{(i)}} p_{z}^{(i)} z
$$

for some integers $p_{z}^{(i)} \geqslant 0$. Put

$$
p:=\sum_{i=1}^{n} \sum_{z \in A \cap S_{(i)}} p_{z}^{(i)} .
$$

Then we will prove by induction on $p$ that $x \in S$. Of course, we may assume that

$$
\sum_{z \in A \cap S_{(i)}} p_{z}^{(i)}>0
$$

for every $i \in[1, n]$. From this it follows that $p \geqslant n$. If $p=n, y^{(i)}=z$ for some $z \in A \cap S_{(i)}$. Therefore, one can find a minimal generic subset $B$ of $A$ such that $x+z \in B$ for all $z \in B$, whence $x \in S_{A}=S$. If $p>n$, there exists an integer $j \in[1, n]$ such that

$$
\sum_{z \in A \cap S_{(i)}} p_{z}^{(j)}>1
$$


Fix an element $z^{\prime} \in A \cap S_{(i)}$ such that $p_{z^{\prime}}^{(j)}>0$. Consider the element $x^{\prime}:=x+$ $y^{(j)}-z^{\prime}$. Then $x^{\prime}+z^{\prime} \in S, x^{\prime}+y^{(i)} \in S$ for $i \neq j$. Since

$$
\sum_{i \neq j} \sum_{z \in A \cap S_{(i)}} p_{z}^{(i)}+1 \leqslant p-1,
$$

$x^{\prime} \in S$ by the induction hypothesis. Now, one can replace $y^{(j)}$ by the element $y^{(j)}-z^{\prime}$. Then, again by the induction hypothesis, we can conclude that $x \in S$.

As an example for Lemma 4.3, consider the simplicial case of [5 and 25]. Recall that an affine semigroup $S$ is called simplicial if $C_{S}$ can be spanned by $r$ elements $e_{1}, \ldots, e_{r}$ of $S$. Algebraically, this means that $k[S]$ has a homogeneous system of parameters of the form $t^{e_{1}}, \ldots, t^{e_{r}}$. Since $C_{S}$ is $r$-dimensional, we can conclude that $C_{S}$ has exactly $r$ extreme rays passing through $e_{1}, \ldots, e_{r}$. Thus, $C_{S}$ has only $r$ $(r-1)$-dimensional faces and each of them is spanned by $r-1$ elements of the set $A:=\left\{e_{1}, \ldots, e_{r}\right\}$. As a consequence, the minimal generic subsets of $A$ are exactly the subsets of two elements. From this it follows that

$$
S_{A}=\left\{x \in G ; x+e_{i} \in S, x+e_{j} \in S \text { for some } i \neq j\right\} .
$$

Corollary 4.4 [5, Theorem 5.1, 25, Theorem 6.4]. Let $S$ be a simplicial affine semigroup. Let $e_{1}, \ldots, e_{r}$ be elements of $S$ which span $C_{S}$. Then $k[S]$ is Cohen-Macaulay (resp. Gorenstein) if and only if

$$
\left\{x \in G ; x+e_{i} \in S, x+e_{j} \in S \text { for some } i \neq j\right\}=S
$$

(resp. $G_{[1, r]}=x-S$ for some $x \in G$ ).

Proof. By Lemma 4.2, one only needs to show that condition (ii) of Theorem 4.1 is satisfied. Let $J$ be an arbitrary proper subset of $[1, r]$. Since the intersection of the faces $F_{i}, i \in J$, contains some element of $\left\{e_{1}, \ldots, e_{r}\right\}, \pi_{J}$ is the simplicial complex of all nonempty subsets of $J$, whence acyclic.

To check condition (ii) of Theorem 4.1 one can also use the artinian property of local cohomology modules [8].

LEMmA 4.5. $\pi_{J}$ is acyclic if $\sup \left\{x_{i} ; x \in G_{J}, i=1, \ldots, n\right\}=\infty$.

Proof. By Theorem 3.3, if $\tilde{H}_{q-2}\left(\pi_{J} ; k\right) \neq 0$ for some $q$, then $\left[H_{\underline{m}}^{q}\left(k\left[S^{\prime}\right]\right)\right]_{x} \neq 0$ for all $x \in G_{J}$. Therefore, if $\sup \left\{x_{i} ; x \in G_{J}, i=1, \ldots, n\right\}=\infty$, one can conclude that $H_{\underline{m}}^{q}\left(k\left[S^{\prime}\right]\right)$ is not artinian, a contradiction.

The following lemma will be used many times in our further investigation.

LEMMA 4.6. Let $S$ be a standard affine semigroup and $J$ a subset of $[1, n]$ such that there exists an element $y \in G_{J}$ with $y_{j}<0$ for all $j \in J$. Then $\sup \left\{x_{i} ; x \in G_{J}\right\}=\infty$ for all $i \notin J$.

PRoOF. By the definition of standard affine semigroups one can find an element $z \in S$ such that $z_{i}>0$ for all $i \in[1, n]$. For any positive integer $p$, choose a positive integer $q$ such that $q y_{j}+p z_{j}<0$ for all $j \in J$. Then $q y+p z \notin S_{j}$ for all $j \in J$. For $i \notin J, q y+p z \in S_{i}$ because $y \in S_{i}$. Therefore, $q y+p z \in G_{J}$. Since $y_{i} \geqslant 0$, $\sup \left\{q y_{i}+p z_{i} ; p>0\right\}=\infty$. 
Now, from Theorem 4.1 and Lemma 4.6 one can easily derive Hochster's result on the Cohen-Macaulayness of normal affine semigroup rings. Recall that an affine semigroup $S$ is called normal if $\bar{S}=S$, where

$$
\bar{S}:=\{x \in G ; p x \in S \text { for some } p>0\} .
$$

Corollary 4.7 [11, Theorem 1, 25, TheORem 6.7]. Let $S$ be a normal affine semigroup. Then $k[S]$ is Cohen-Macaulay. Moreover, $k[S]$ is Gorenstein if and only if $G_{[1, m]}=x-S$ for some $x \in G$.

Proof. Since $S \subseteq S^{\prime} \subseteq \bar{S}=S, S^{\prime}=S$. It remains to show condition (ii) of Theorem 4.1. For this, we may assume that $S$ is standard and set $S_{i}=S-S_{(i)}$, $i=1, \ldots, n(\S 1)$. By Lemmas 4.5 and 4.6, it is sufficient to show that $S_{i}=\{x \in G$; $\left.x_{i} \geqslant 0\right\}$ because this implies

$$
G_{J} \subseteq\left\{x \in G ; x_{i}<0 \text { for all } i \in J\right\}
$$

for all subsets $J$ of $[1, n]$. Let $x$ be an arbitrary element of $G$ with $x_{i} \geqslant 0$. Choose an element $y \in S_{(i)}$ with $y_{i}>0$ for all $j \neq i$ (this is possible because $S_{(i)} \neq S_{(j)}$ ). Then $x_{j}+p y_{j} \geqslant 0$ for $p$ sufficiently large. Thus, $x+p y \in G \cap N^{n}=\bar{S}=S$. It follows that $x \in S-S_{(i)}=S_{i}$. Hence we get the above formula for $S_{i}$.

Theorem 4.1 can be also used to study the problem when a blowing-up extension $E_{\lambda}$ of $S$ (see definition in \$1) yields a Cohen-Macaulay (resp. Gorenstein) ring $k\left[E_{\lambda}\right]$.

LeMma 4.8. Let $E_{\lambda}$ be a blowing-up extension of $S$. Then $k\left[E_{\lambda}\right]$ is Cohen-Macaulay (resp. Gorenstein) if and only if the following conditions are satisfied:

(i) $S^{\prime}=S\left(\right.$ resp. $G_{[1, m]}=x-S$ for some $x \in G$ with $\left.\lambda(x)=-2\right)$.

(ii) For every nonempty proper subset $J$ of $[1, m], \lambda\left(G_{J}\right) \subseteq\{-1\}$ or $\tilde{H}_{q}\left(\pi_{J} ; k\right)=0$ for all $q<r-2$.

(iii) $\lambda\left(G_{[1, m]}\right) \cap N=\varnothing$.

Proof. Set $E=E_{\lambda}$. By Lemma $1.1, E^{\prime}=E$ if and only if $S^{\prime}=S$. So, for the Cohen-Macaulay case, one need only prove that condition (ii) of Theorem 4.1 formulated for $E$ is equivalent to the above conditions (ii) and (iii). First, we note that the convex cone $C_{E}$ is $(r+1)$-dimensional and that $C_{E}$ has $m+2 r$-dimensional faces $P_{1}, \ldots, P_{m+2}$ as described in the proof of Lemma 1.1. Let $I$ be an arbitrary nonempty proper subset of $[1, m+2]$. Let $\psi_{I}$ denote the simplicial complex of nonempty subsets $I^{\prime}$ of $I$ such that $\bigcap_{i \in I^{\prime}} E \cap P_{i} \neq(0)$ and set

$$
G_{I}(E)=\bigcap_{j \notin I} E_{j} \backslash \bigcup_{i \in I} E_{i} .
$$

Put $J=I \cap[1, m]$. Then we have to distinguish three cases:

(1) $I=J$. Then $\psi_{I}=\pi_{J}$, and

$$
G_{I}(E)=\left\{(x, p) \in Z^{n+1} ; x \in G_{J} \text { and } \lambda(x) \geqslant p \geqslant 0\right\}
$$

(this follows from the presentations of $P_{1}, \ldots, P_{m}, E_{1}, \ldots, E_{m}$ in the proof of Lemma 1.1). Obviously, $G_{I}(E)=\varnothing$ if and only if $\lambda\left(G_{J}\right) \cap N=\varnothing$.

(2) $I=J \cup\{m+1\}$ or $I=J \cup\{m+2\}$. Then $\psi_{I}$ is the join of $\pi_{J}$ with the point $\{m+1\}$ or $\{m+2\}$. Therefore, $\psi_{I}$ as the cone of $\pi_{J}$ is acyclic [24]. 
(3) $I=J \cup\{m+1, m+2\}$. Then $J$ is a proper subset of [1,m]. Since $\psi_{I}$ is the join of $\pi_{J}$ with the complex $\{\{m+1\},\{m+2\}\}, \tilde{H}_{q}\left(\psi_{I} ; k\right)=\tilde{H}_{q}\left(\pi_{J} ; k\right)$ for all $q$. Moreover, since

$$
G_{I}(E)=\left\{(x, p) \in Z^{n+1} ; x \in G_{J} \text { and } \lambda(x)<p<0\right\},
$$

$G_{I}(E)=\varnothing$ if and only if $\lambda\left(G_{J}\right) \subseteq\{-1,0,1, \ldots\}$. From all these facts, one can conclude that condition (ii) of Theorem 4.1 formulated for $E$ is equivalent to (ii) and (iii), where (iii) comes from the condition $G_{[1, m]}(E)=\varnothing$ because $\psi_{[1, m]}=\pi_{[1, m]}$ is not acyclic (Corollary 3.6(i)). Concerning the Gorenstein case, one need only show that $G_{[1, m+2]}(E)=(x, p)-E$ for some $(x, p) \in G(E)$ if and only if $G_{[1, m]}=x-S$ for some $x \in G$ with $\lambda(x)=-2$. Since

$$
G_{[1, m+2]}(E)=\left\{(y, q) \in Z^{n+1} ; y \in G_{[1, m]} \text { and } \lambda(y)<q<0\right\},
$$

the first condition is satisfied if and only if there exist $x \in G$ and $p \in Z$ such that

(4) $G_{[1, m]}=x-S$,

(5) $\lambda(x-y) \geqslant p-q \geqslant 0$ for any $y \in G_{[1, m]}$ and $q \in Z$ with $\lambda(y)<q<0$,

(6) $\lambda(x-z)<p-s<0$ for any $z \in S$ and $s \geqslant 0$ with $\lambda(z) \geqslant s$.

Obviously, (5) (resp. (6)) can be written as $\lambda(x) \geqslant p-1$ and $p \geqslant-1$ (resp. $\lambda(x)<p<0)$. Hence (5) and (6) are satisfied iff $\lambda(x)=-2$ and $p=-1$. The proof is now complete.

EXAMPLE 4.9. $k[S]$ need not be a Cohen-Macaulay ring if $k\left[E_{\lambda}\right]$ is CohenMacaulay. Let $S$ be the affine semigroup of Example 3.9. Let $\lambda$ be the linear functional $x \rightarrow x_{1}+2 x_{2}$ from $Q^{4}$ to $Q$. We have seen that $k[S]$ is a non-CohenMacaulay ring with $S^{\prime}=S$ and that, for an arbitrary nonempty proper subset $J$ of $[1,4]$ with $J \neq\{1,2\}, G_{J}=\varnothing$ or $\tilde{H}_{q}\left(\pi_{J} ; k\right)=0$ for all $q$. If $J=\{1,2\}, G_{J}=$ $\{(1,-1,0,0)\}$ and $\lambda\left(G_{J}\right)=\{-1\}$. Since $G_{[1,4]}=\left\{x \in Q^{4} ; x_{1}<0\right.$ or $x_{1}=1, x_{i}<0$, $i=2,3,4$, and $x_{1}+x_{2}=3 x_{3}+x_{4}=3 p$ for some $\left.p \in Z\right\}, \lambda\left(G_{[1,4]}\right) \cap N=\varnothing$. Therefore, by the above criterion, $k\left[E_{\lambda}\right]$ is Cohen-Macaulay. It should be noted that if $\lambda$ is the linear functional $x \rightarrow x_{1}+x_{2}, k\left[E_{\lambda}\right]$ is not Cohen-Macaulay.

We conclude $\S 4$ with some observation on the Buchsbaumness of affine semigroup rings. Recall that a local ring $A$ with maximal ideal $\underline{a}$ is called a Buchsbaum ring if for every system of parameters $a_{1}, \ldots, a_{d}$ of $A$,

$$
\left(a_{1}, \ldots, a_{i}\right): \underline{a}=\left(a_{1}, \ldots, a_{i}\right): \underline{a}^{2}
$$

for $i=0, \ldots, d-1[27,28]$. Of course, Buchsbaum rings are natural generalizations of local Cohen-Macaulay rings.

Goto [3] claimed that $k[S]_{m}$ is a Buchsbaum ring if and only if $S^{\prime}+(S \backslash(0)) \subseteq S$ (the proof uses [6, Theorem 3.3.3]). This result is also false by the following example.

EXAMPLE 4.10. Let $S$ be the affine semigroup in $N^{3}$ generated by the elements $(3,0,0),(2,1,0),(0,3,0),(0,3,1),(3,0,1),(4,2,1),(5,1,2),(3,1,2)$. Then it is not hard to see that $S^{\prime}$ is the affine semigroup $E_{\lambda}$ of Example 1.2 and that $S^{\prime}+(S \backslash(0))$ $\subseteq S$. But $k[S]_{\underline{m}}$ is not a Buchsbaum ring because $t_{1}^{3}, t_{2}^{3} t_{3}$ form a part of a system of parameters of $k[S]_{\underline{m}}$ with

$$
t_{1}^{4} t_{2}^{2} t_{3} \underline{m}^{2} \subseteq\left(t_{1}^{3}, t_{2}^{3} t_{3}\right), \quad t_{1}^{4} t_{2}^{2} t_{3} \cdot t_{1}^{3} t_{3} \notin\left(t_{1}^{3}, t_{2}^{3} t_{3}\right) .
$$


It is interesting to note that from the formula for local cohomology modules of $k$ [ $\left.S^{\prime}\right]$ (given in Example 3.9 for another but isomorphic affine semigroup) one can even conclude that $k\left[S^{\prime}\right]_{\underline{m}}$ is a Buchsbaum ring [28, Corollary 1.1].

LEMMA 4.11. $S^{\prime}+(S \backslash(0)) \subseteq S$ if $k[S]_{\underline{m}}$ is a Buchsbaum ring. The converse holds if $k\left[S^{\prime}\right]$ is Cohen-Macaulay.

Proof. If $k[S]_{\underline{m}}$ is a Buchsbaum ring, $k[S]_{\underline{p}}$ is Cohen-Macaulay for all graded prime ideals $\underline{p} \neq \underline{m}$ of $k[S][2,(3.5)]$. Thus, by Corollary 2.3, $\operatorname{dim} k\left[S^{\prime} \backslash S\right]=0$. By Corollary 3.4, this implies $H_{\underline{m}}^{1}(k[S]) \cong k\left[S^{\prime} \backslash S\right]\left(S^{\prime \prime}=S^{\prime}\right)$. Therefore, by [28], $k\left[S^{\prime} \backslash S\right]$ is annihilated by $\underline{m}$, i.e. $S^{\prime}+(S \backslash(0)) \subseteq S$. Conversely, if $S^{\prime}+(S \backslash(0)) \subseteq$ $S$ and if $k\left[S^{\prime}\right]$ is Cohen-Macaulay, then $\underline{m} k\left[S^{\prime} \backslash S\right]=0$ and $H_{\underline{m}}^{q}\left(k\left[S^{\prime}\right]\right)=0$ for all q. Therefore, from the exact sequence

$$
0 \rightarrow k[S] \rightarrow k\left[S^{\prime}\right] \rightarrow k\left[S^{\prime} \backslash S\right] \rightarrow 0
$$

one can derive that

$$
H_{\underline{m}}^{q}(k[S])= \begin{cases}0 & \text { if } q \neq 1, r, \\ k\left[S^{\prime} \backslash S\right] & \text { if } q=1 .\end{cases}
$$

Hence by [22, Corollary 1.1], $k[S]_{\underline{m}}$ is a Buchsbaum ring.

REMARK. If $S$ is a simplicial affine semigroup, $k\left[S^{\prime}\right]$ is always Cohen-Macaulay because $\left(S^{\prime}\right)^{\prime}=S^{\prime}$. In this case, from Lemma 4.11 one can derive a simple criterion for $k[S]_{\underline{m}}$ to be Buchsbaum which is similar to Corollary 4.4 [29, Lemma 3]. It remains an open question whether there exists a general criterion for $k[S]_{\underline{m}}$ being Buchsbaum in terms of $S$.

5. Dependence upon the characteristic of $k$. The investigation of $\S 3$ leads us to suspect that the vanishing of local cohomology modules of $k[S]$ may depend upon the characteristic of $k$. We can actually confirm this by the following result.

THEOREM 5.1. For every finite simplicial complex $\Delta$ with $r$ vertices, there exists an affine semigroup $S$ with $\operatorname{rank}_{Z} G=r$ such that $H_{\underline{m}}^{q}(k[S])=\tilde{H}_{q-2}(\Delta ; k)$ for $q=$ $0, \ldots, r-1$.

The proof of Theorem 5.1 is based on the following observations.

LEMMA 5.2. Let $L$ be a subgroup of $Z^{n}$ such that for every $i=1, \ldots, n$, there exists an element $x \in L$ with $x_{i}=0, x_{j}>0$ for all $j \neq i$. Let $s_{1}, \ldots, s_{n}$ be arbitrary nonnegative integers. Put

$$
S=\left\{x \in L \cap N^{n} ; x_{i}=0 \text { or } x_{i}>s_{i}, i=1, \ldots, n\right\} .
$$

Then $S$ is a standard affine semigroup with $G=L, S^{\prime}=S$.

Proof. First, we have to check the conditions of standard affine semigroups given in $\$ 1$. Choose an element $x \in S$ such that $x_{i}>0$ for all $i=1, \ldots, n$. If $e_{1}, \ldots, e_{s}$ generate $L$, so do $x, e_{1}+p x, \ldots, e_{s}+p x$ for every integer $p$. Since $e_{1}+p x, \ldots, e_{s}$ $+p x \in S$ for $p$ sufficiently large, $G=G(S)=L$. From this it follows that $\bar{S}=G$ $\cap N^{n}$. By the assumption on $L$, we also have $S_{(i)} \neq S_{(j)}$ for $i \neq j$. Moreover, using the same argument as above, we can show that $G\left(S_{(i)}\right)=G\left(L \cap H_{i}\right)$, where $H_{i}$ 
denotes the hyperplane $x_{i}=0, i=1, \ldots, n$. Let $W$ denote the linear space spanned by $L$ in $Q^{n}$. Since $L \cap H_{i}$ spans the space $W \cap H_{i}$ and $W \nsubseteq H_{i}$,

$$
\operatorname{rank}_{Z} G\left(S_{(i)}\right)=\operatorname{dim}_{Q} W \cap H_{i}=\operatorname{dim}_{Q} W-1=r-1 .
$$

Therefore, we can conclude that $S$ is a standard affine semigroup. Now we can set $S_{i}=S-S_{(i)}$ and get

$$
S_{i}=\left\{x \in L ; x_{i}=0 \text { or } x_{i}>s_{i}\right\},
$$

$i=1, \ldots, n$. From this we see that $S^{\prime}=\bigcap_{i=1}^{n} S_{i}=S$.

LEMMA 5.2 will be used to give a large class of affine semigroup $S$ with $S^{\prime}=S$ such that $\Delta=\pi_{J}$ for some $J \subset[1, n]$.

Let $\Delta_{[1, r]}$ be the simplicial complex of all nonempty subsets of $[1, r]$. Consider $\Delta$ as a subcomplex of $\Delta_{[1, r]}$. Suppose that $\Delta_{[1, r]} \backslash \Delta$ has $s$ minimal simplexes. Then we will denote these simplexes by $I_{r+1}, \ldots, I_{n}, n=r+s$.

LEMMA 5.3. Suppose that $\Delta \neq \Delta_{[1, r]}$. Let

$$
\sum_{j=1}^{r} a_{i j} X_{j}=a_{i} X_{i}, \quad i=r+1, \ldots, n,
$$

be a system of linear equations with integral coefficients such that $a_{i}>0, I_{i}=\{j \in$ $\left.[1, r] ; a_{i j} \geqslant 0\right\}$, and, if $a_{i j} \geqslant 0, a_{i j}+\sum_{t \notin I_{i}} a_{i t}>0$. Let $L$ be a subgroup of the additive group of integral solutions of $(*)$ with $\operatorname{rank}_{Z} L=r$. Let $s_{1}, \ldots, s_{n}$ be arbitrary integers. Put

$$
S=\left\{x \in L \cap N^{n} ; x_{i}=0 \text { or } x_{i}>s_{i}, i=1, \ldots, n\right\} .
$$

Then $S$ is a standard affine semigroup with $G=L, S^{\prime}=S$, and $\pi_{[1, r]}=\Delta$.

Proof. Let $I$ be an arbitrary subset of $[1, r]$. If $I \in \Delta, I \nsupseteq I_{i}$ for all $i=r+$ $1, \ldots, n$. Hence $\sum_{j \notin I} a_{i j}>0$ by the assumption. Set $a=a_{r+1} \cdots a_{n}$. Consider the solution $y$ of $(*)$ with

$$
y_{i}= \begin{cases}0 & \text { if } i \in I, \\ a & \text { if } i \in[1, r] \backslash I, \\ \left(a / a_{i}\right) \sum_{j \notin I} a_{i j} & \text { if } i \in[r+1, n],\end{cases}
$$

where $[r+1, n]$ denotes the set of integers $r+1, \ldots, n$. Since $\operatorname{rank}_{Z} L=r$ is the rank of the group of integral solutions of $(*)$ over $Z$, all sufficiently large multiples of $y$ must belong to $L$ and, therefore, to $S$. From this it follows that $\bigcap_{i \in I} S_{(i)} \neq(0)$. If $I \notin \Delta, I \supseteq I_{i}$ for some $i \in[r+1, n]$. In this case, if $x \in S_{(j)}$ for all $j \in I$, from the equation

$$
\sum_{j=1}^{r} a_{i j} x_{j}=a_{i} x_{i}
$$

we can conclude that $x_{j}=0$ for all $j \in[1, r], j=i$ and, therefore, for all $j \in[1, n]$ by using the other equations of $(*)$. So we have proved that

$$
\Delta=\left\{I \subset[1, r] ; \bigcap_{i \in I} S_{(i)} \neq(0)\right\} .
$$


It remains to show that $S$ is a standard affine semigroup with $L=G, S^{\prime}=S$. For this we need only show that $L$ satisfies the condition of Lemma 5.2. First, for every $i \in[1, r]$, one can consider $i$ as a simplex of $\Delta$. Hence, as shown above, $L$ has an element $x$ with $x_{i}=0, x_{j}>0$ for all $j \neq i$. For $i \in[r+1, n]$, put

$$
b=-\sum_{j \notin I_{i}} a_{i j}, \quad c=\sum_{j \in I_{i}} a_{i j} .
$$

Then $b$ and $c$ are positive integers with $b<c$. Consider the integral solution $z$ of $(*)$ with

$$
z_{t}= \begin{cases}a b & \text { if } t \in I_{i}, \\ a c & \text { if } t \in[1, r] \backslash I_{i}, \\ \left(a / a_{t}\right)\left(\sum_{j \in I_{i}} a_{t j} b+\sum_{j \notin I_{i}} a_{t j} c\right) & \text { if } t \in[r+1, n] .\end{cases}
$$

Obviously, $z_{i}=0$. For $t \neq i, t \in[r+1, n]$,

$$
z_{t} \geqslant \frac{a b}{a_{t}}\left(a_{t u}+\sum_{j \notin I_{t}} a_{t j}\right)>0,
$$

where $u$ is some element of $I_{t} \backslash I_{i}$. Therefore, we can also conclude that $L$ has an element $x$ with $x_{i}=0, x_{j}>0$ for all $j \neq i$.

Proof of TheOrem 5.1. If $\Delta$ is acyclic, one only need to set $S=N^{r}$. Assume that $\Delta$ is not acylic. Then $\Delta \neq \Delta_{[1, r]}$. Consider the following linear equations

$$
\sum_{j \in I_{i}} r X_{j}-\sum_{j \in[1, r] \backslash I_{i}} X_{j}=\left(r n_{i}-r+n_{i}\right) X_{i},
$$

$i=r+1, \ldots, n$, where $n_{i}=\# I_{i}$. Let $L$ be the group of integral solutions $x$ of the system of the above equations with $x_{i} \equiv x_{j}$ modulo 2 for all $i, j=1, \ldots, n$. Obviously, $\operatorname{rank}_{Z} L=r$. Put

$$
S=\left\{x \in L \cap N^{n} ; x_{i} \neq 1 \text { for all } i \in[1, r]\right\} .
$$

By Lemma 5.3, $S$ is a standard affine semigroup with $G=L, S^{\prime}=S$, and $\pi_{[1, r]}=\Delta$. Therefore, by Corollary 3.7, one has to show that $G_{[1, r]}$ contains only one element and that for every nonempty proper subset $J \neq[1, r]$ of $[1, n], G_{J}=\varnothing$ or $\pi_{J}$ is acyclic. Note that

$$
S_{i}= \begin{cases}\left\{x \in L ; x_{i} \geqslant 0 \text { and } x_{i} \neq 1\right\} & \text { if } i \in[1, r], \\ \left\{x \in L ; x_{i} \geqslant 0\right\} & \text { if } i \in[r+1, n] .\end{cases}
$$

Then one can easily check that $(1, \ldots, 1) \in G_{[1, r]}$. Therefore, it is sufficient to prove the following claim.

Claim. Let $J$ be an arbitrary nonempty proper subset of $[1, n]$. Suppose that $G_{J}$ contains an element $x \neq(1, \ldots, 1)$. Then $\pi_{J}$ is acyclic.

Assume the contrary. Then, for all $i \in[1, n], \sup \left\{y_{i} ; y \in G_{J}\right\}<\infty$ by Lemma 4.5. By Lemma $4.6, x_{j} \geqslant 0$ for some $j \in J$. Note that

$$
G_{J}=\bigcap_{i \notin J} S_{i} \backslash \bigcup_{i \in J} S_{i}
$$


Then, by the above formula for $S_{i}$, this is only possible if $j \in[1, r]$ and $x_{j}=1$. Therefore, for all $i \in[1, n], x_{i} \neq 0$ because $x_{i} \equiv 1$ modulo 2. Let $p$ be an arbitrary positive integer. Then $(p+1) x_{i}-p \geqslant 0$ iff $x_{i} \geqslant 0$ and $(p+1) x_{i}-p=1$ iff $x_{i}=1, i=1, \ldots, n$. Therefore $(p+1) x-p(1, \ldots, 1) \in S_{i}$ if and only if $x \in S_{i}$. So, like $x \in G_{J},(p+1) x-p(1, \ldots, 1) \in G_{J}$. Hence, by the above assumption, $(p+1) x_{i}-p$ cannot be arbitrarily large. Hence either $x_{i}=1$ or $x_{i}<0$. As a consequence, $x \notin S_{i}$ for all $i \in[1, r]$, which implies $J \supseteq[1, r]$. Put

$$
I:=\left\{i \in[1, r] ; x_{i}=1\right\}, \quad a:=2 \prod_{i=1}^{r}\left(r n_{i}-r+n_{i}\right) .
$$

Consider the element $y \in L$ with

$$
y_{i}= \begin{cases}0 & \text { if } i \in I, \\ a x_{i} & \text { if } i \in[1, r] \backslash I, \\ a x_{i}-a(r b-c) /\left(r n_{i}-r+n_{i}\right) & \text { if } i \in[r+1, n],\end{cases}
$$

where $b:=\#\left(I \cap I_{i}\right)$ and $c:=\#\left(I \backslash I_{i}\right)$. We shall see that for all $i \in[1, n]$, $x+p y \in S_{i}$ if and only if $x \in S_{i}$. For $i \in[1, r]$, that is immediate. For $i \in[r+1, n]$, we have to show that $x_{i}+p y_{i} \geqslant 0$ if and only if $x_{i} \geqslant 0$. If $x_{i}<0$,

$$
y_{i}=a\left(x_{i}+1\right)-\frac{a\left[r b+\left(r-n_{i}-c\right)+2 n_{i}+r\left(n_{i}-2\right)\right]}{r n_{i}-r+n_{i}}<0
$$

because $r \geqslant n_{i}+c$ and $n_{i} \geqslant 2$ ( $\Delta$ is not acyclic). Hence $x_{i}+p y_{i}<0$. If $x_{i} \geqslant 0$, then $x_{i}=1$. In this case, if

$$
y_{i}=\frac{a\left[r\left(n_{i}-1-b\right)+c+n_{i}\right]}{r n_{i}-r+n_{i}} \leqslant 0
$$

we must have $b=n_{i}$, i.e. $x_{j}=1$ for all $j \in I_{i}$. Now, from the equation associated with $I_{i}$ we get

$$
\sum_{j \in\left[1, r \backslash \backslash I_{i}\right.} x_{j}=r-n_{i} .
$$

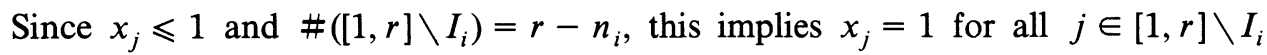
and therefore for all $j \in[r+1, n]$ by using the other equations. Hence $x=$ $(1, \ldots, 1)$, a contradiction. Thus, we must have $y_{i}>0$ and, therefore, $x_{i}+p y_{i} \geqslant 0$. So we have proved that $x+p y \in S_{i}$ if and only if $x \in S_{i}$ for all $i \in[1, n]$. Thus $x+p y \in G_{J}$. Hence $x_{i}+p y_{i}$ cannot be arbitrarily large. Hence $y_{i} \leqslant 0$. For $i \in$ $[r+1, n]$, this implies $x_{i}<0$ by the above analysis. From this it follows that $x \notin S_{i}$ for $i \in[r+1, n]$. Hence $J \supseteq[r+1, n]$. Summing up, we get $J=[1, n]$, a contradiction. So we have proved the claim and, therefore, Theorem 5.1.

Now we will illustrate Theorem 5.1 by giving an affine semigroup ring $k[S]$ whose Cohen-Macaulayness depends upon the characteristic of $k$.

EXAMPLe 5.4. Let $\Delta$ be the simplicial complex associated with the minimal triangulation of the projective plane: 


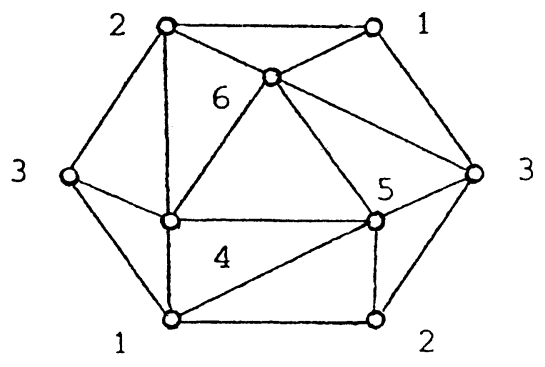

Then $\Delta$ can be considered as a subcomplex of the simplicial complex $\Delta_{[1,6]}$ of nonempty subsets of $[1,6]$. Obviously, $\Delta_{[1,6]} \backslash \Delta$ has the following 10 minimal simplexes: $\{1,2,3\},\{1,2,4\},\{1,3,5\},\{1,4,6\},\{1,5,6\},\{2,3,6\},\{2,4,5\},\{2,5,6\}$, $\{3,4,5\},\{3,4,6\}$. Consider the system of linear equations

$$
\begin{aligned}
6\left(X_{1}+X_{2}+X_{3}\right) & =X_{4}+X_{5}+X_{6}+15 X_{7}, \\
6\left(X_{1}+X_{2}+X_{4}\right) & =X_{3}+X_{5}+X_{6}+15 X_{8} \\
6\left(X_{1}+X_{3}+X_{5}\right) & =X_{2}+X_{4}+X_{6}+15 X_{9}, \\
6\left(X_{1}+X_{4}+X_{6}\right) & =X_{2}+X_{3}+X_{5}+15 X_{10} \\
& \vdots \\
6\left(X_{3}+X_{4}+X_{6}\right) & =X_{1}+X_{2}+X_{5}+15 X_{16} .
\end{aligned}
$$

Let $S$ be the affine semigroup of solutions $x \in N^{16}$ of this system of linear equations with $x_{i} \equiv x_{j}$ modulo 2 for all $i, j \in[1,16]$ and $x_{i} \neq 1$ for all $i \in[1,6]$. Then, by the proof of Theorem $5.1, \operatorname{dim} k[S]=6$ and $H_{\underline{m}}^{q}(k[S])=\tilde{H}_{q-2}(\Delta ; k)$ for $q=0, \ldots, 5$. Since

$$
\tilde{H}_{q-2}(\Delta ; Z)= \begin{cases}0 & \text { if } q \neq 3, \\ Z_{2} & \text { if } q=3,\end{cases}
$$

one can conclude that $k[S]$ is a Cohen-Macaulay ring if and only if $\operatorname{char}(k) \neq 2$, cf. [20] for a similar result.

Another consequence of Theorem 5.1 is the following result concerning the existence of a unique minimal Macaulayfication of an affine semigroup ring.

COROLlaRY 5.5. There exist non-Cohen-Macaulay affine semigroup rings $k[S]$ which are the intersections of all Cohen-Macaulay rings $k[E]$ with $E \supseteq S$.

Proof. Let $S$ be as in the proof of Theorem 5.1. Then $S$ is the intersection of the affine semigroups $\left\{x \in L \cap N^{n} ; x_{i} \neq 1\right\}, i=1, \ldots, n$. We claim that their semigroup rings are Cohen-Macaulay. Of course, it suffices to consider the case

$$
E=\left\{x \in L \cap N^{n} ; x_{1} \neq 1\right\} \text {. }
$$

First, it is easily seen that $E$ is a standard affine semigroup with $E^{\prime}=E$ and

$$
\begin{aligned}
& E_{1}=\left\{x \in G ; x_{1} \geqslant 0 \text { and } x_{1} \neq 1\right\}, \\
& E_{i}=\left\{x \in G ; x_{i} \geqslant 0\right\}, \quad i \in[2, n] .
\end{aligned}
$$

For every nonempty proper subset $J$ of $[1, n]$, set

$$
G_{J}(E):=\bigcap_{i \in J} E_{i} \backslash \bigcup_{i \notin J} E_{i} \text {. }
$$


Then, by Theorem 4.1 and Lemma 4.5 , one only needs to show that $\sup \left\{x_{i}\right.$; $\left.x \in G_{J}(E)\right\}=\infty$ for some $i \in[1, n]$ if $G_{J}(E) \neq \varnothing$. Assume the contrary. Let $x$ be an arbitrary element of $G_{J}(E)$. By Lemma 4.6, one has $x_{1}=1$. From this it follows that $1 \in J$. Hence $x_{i} \neq 0$ for all $i \in[1, n]$ because $x_{i} \equiv 1$ modulo 2. Now, consider the element $y \in L$ with

$$
y_{i}:= \begin{cases}0 & \text { if } i=1, \\ a x_{i} & \text { if } i \in[2, r], \\ a x_{i}-a r /\left(r n_{i}-r+n_{i}\right) & \text { if } 1 \in I_{i}, i \in[r+1, n], \\ a x_{i}+a /\left(r n_{i}-r+n_{i}\right) & \text { if } 1 \notin I_{i}, i \in[r+1, n] .\end{cases}
$$

It is not hard to check that for every positive integer $p$ and $i \in[1, n], x+p y \in E_{i}$ if and only if $x \in E_{i}$. This means that $x+m y \in G_{J}(E)$. Hence $x_{i}+m y_{i}$ cannot be arbitrarily large. Hence $y_{i} \leqslant 0$. Thus, $x_{i}<0$ or, equivalently, $x \notin E_{i}$ for all $i \in[2, n]$. So one would obtain $J=[1, n]$, a contradiction.

\section{REFERENCES}

1. G. E. Cooke and R. L. Finney, Homology of cell complexes, Math. Notes, Princeton Univ. Press, 1967.

2. N. T. Cuong, P. Schenzel and N. V. Trung, Verallgemeinerte Cohen-Macaulay-Moduln, Math. Nachr. 85 (1978), 57-73.

3. S. Goto, On the Macaulayfication of certain Buchsbaum rings, Nagoya Math. J. 80 (1980), 107-116.

4. S. Goto and Y. Shimoda, On the Rees algebra of Cohen-Macaulay local rings, Commutative Algebra (Analytic Method), Dekker, New York, 1982, pp. 201-231.

5. S. Goto, N. Suzuki and K. Watanabe, On affine semigroups, Japan. J. Math. 2 (1976), 1-12.

6. S. Goto and K. Watanabe, On graded rings. II: $Z^{n}$-graded rings, Tokyo J. Math. 1 (1978), 237-261.

7. W. Gröbner, Über Veronesesche Varietäten und deren Projektionen, Arch. Math. 16 (1965), 257-264.

8. A. Grothendieck (noted by R. Hartshorne), Local cohomology, Lecture Notes in Math., vol. 41, Springer-Verlag, Berlin and New York, 1967.

9. B. Grünbaum, Convex polytopes, Wiley, 1967.

10. T. Hibi, Affine semigroup rings and Hodge algebras. Some recent developments in the theory of commutative rings, Sûrikaisekikenkyûsho Kôkyûroku 484 (1983), 42-51.

11. M. Hochster, Rings of invariants of tori, Cohen-Macaulay rings generated by monomials, and polytopes, Ann. of Math. (2) 96 (1972), 318-337.

12. M. Hochster and J. L. Roberts, Rings of invariants of reductive groups acting on regular rings are Cohen-Macaulay, Adv. in Math. 13 (1974), 115-178.

13. M. Ishida, Toric embeddings and dualizing complexes, Tôhoku Math. J. 32 (1980), 111-146.

14. S. Ikeda, The Cohen-Macaulayness of the Rees algebras of local rings, Nagoya Math. J. 89 (1983), 47-63.

15. G. Kempf, F. Knudsen, D. Mumford and B. Saint-Donat, Toroidal embeddings. I, Lecture Notes in Math. vol. 339, Springer-Verlag, Berlin and New York, 1973.

16. S. Mac Lane, Homology, Springer, 1963.

17. F. S. Macaulay, Algebraic theory of modular systems, Cambridge Tracts in Math., vol. 19, 1916.

18. H. Matsumura, Commutative algebra, Benjamin, 1970.

19. T. Oda, Torus embeddings and applications, Lecture Notes of Tata Institute, Springer, 1982.

20. G. Reisner, Cohen-Macaulay quotient of polynomial rings, Adv. in Math. 21 (1976), 30-49.

21. P. Schenzel, On Veronesean embeddings and projections of Veronesean varieties, Arch. Math. 30 (1978), 391-397.

22. R. Y. Sharp, The Cousin complex for a module over a commutative noetherian ring, Math. Z. 112 (1969), 348-356.

23. Local cohomology and the Cousin complex for a commutative noetherian ring, Math. Z. 112 (1977), 19-22. 
24. E. H. Spanier, Algebraic topology, McGraw-Hill, 1966.

25. R. Stanley, Hilbert functions of graded algebras, Adv. in Math. 28 (1978), 57-73.

26. R. Stanley, Linear diophantine equations and local cohomology, Invent. Math. 68 (1982), 175-193.

27. J. Stückrad and W. Vogel, Eine Verallgemeinerung der Cohen-Macaulay-Ringe und Anwendungen auf ein Problem der Multiplizitätstheorie, J. Math. Kyoto Univ. 13 (1973), 513-528.

28. , Toward a theory of Buchsbaum singularities, Amer. J. Math. 100 (1978), 717-746.

29. N. V. Trung, Classification of the double projections of Veronese varieties, J. Math. Kyoto Univ. 22 (1982), 567-581.

30. __ On projections of one-dimensional Veronese varieties, Math. Nachr. 118 (1984), 47-67.

31. L. T. Hoa, Classification of the triple projections of Veronese varieties, Math. Nachr. (to appear).

INSTitute of Mathematics, Box 631, Bō Hō, HaNoI, Vietnam 